Document downloaded from:

http://hdl.handle.net/10251/122871

This paper must be cited as:

Broatch, A.; Ruiz, S.; Garcia Tiscar, J.; Roig-Villanueva, F. (2018). On the influence of inlet elbow radius on recirculating backflow, whoosh noise and efficiency in turbocharger compressors. Experimental Thermal and Fluid Science. 96:224-233.

https://doi.org/10.1016/j.expthermflusci.2018.03.011

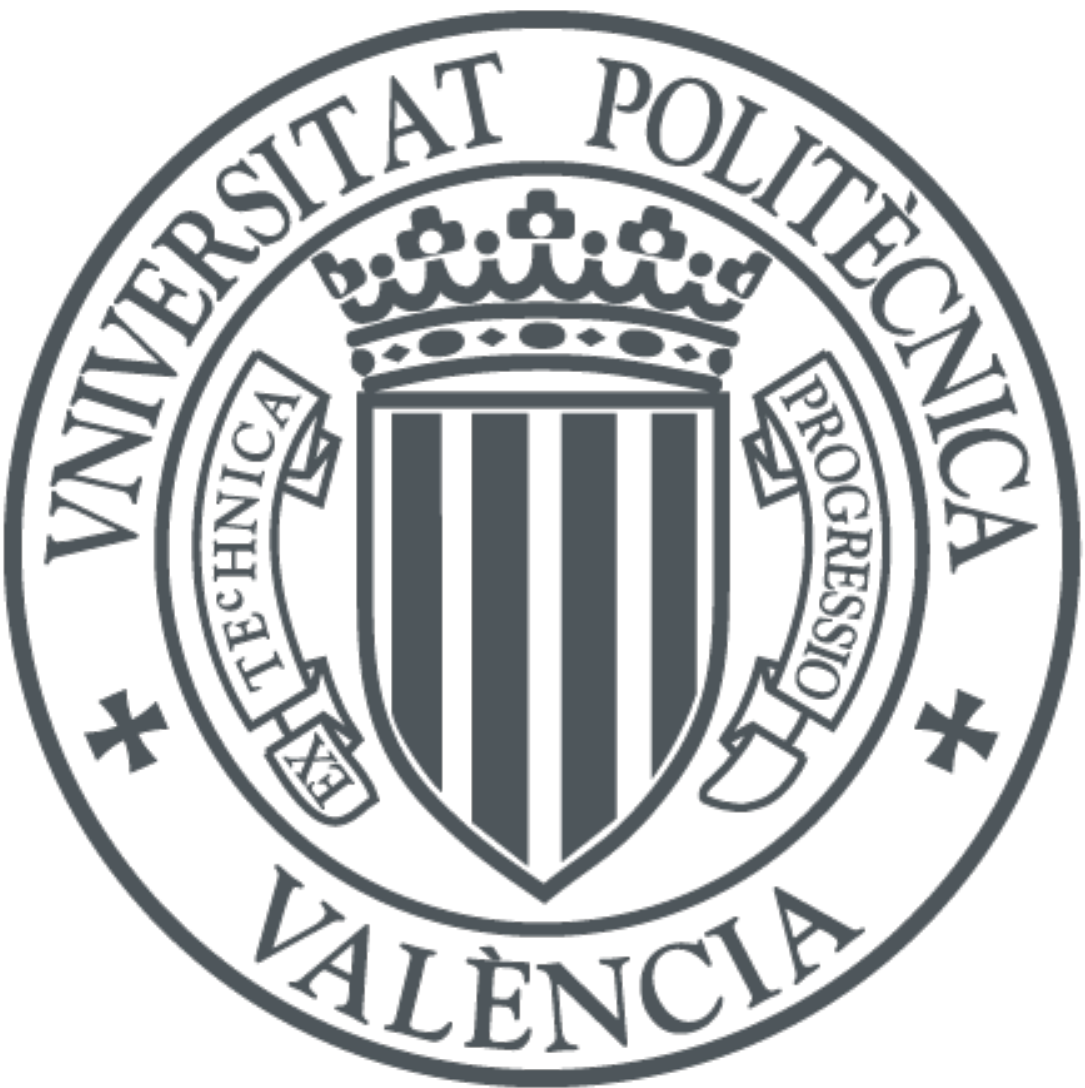

The final publication is available at

http://doi.org/10.1016/j.expthermflusci.2018.03.011

Copyright Elsevier

Additional Information 


\title{
On the influence of inlet elbow radius on recirculating backflow, whoosh noise and efficiency in turbocharger compressors
}

\author{
A. Broatch ${ }^{\mathrm{a}}$, S. Ruiz ${ }^{\mathrm{a}}$, J. García-Tíscar ${ }^{\mathrm{a}, *}$, F. Roig ${ }^{\mathrm{a}}$ \\ ${ }^{a}$ CMT - Motores Térmicos, Universitat Politècnica de València, Camino de Vera, 46022 Valencia, Spain
}

\begin{abstract}
While the influence of inlet geometry on turbocharger compressor behaviour has usually been investigated in terms of performance, surge margin and efficiency, data is scarce regarding the impact of the inlet flow field onto the noise emission. In many applications where tight packaging is required, a $90^{\circ} \mathrm{elbow}$ is placed just upstream of the compressor inducer. This can create a distortion of the incoming flow that affects the turbocharger operation; a distortion that is related to the radius of the elbow. In this experimental investigation three $90^{\circ}$ elbow inlets are tested, measuring the in-duct sound intensity through acoustic beamforming, the spectral signature of the noise, and the distortion of the high temperature backflow typical of partially stalled conditions by means of thermocouple arrays. Results show that a tighter elbow radius not only impacts efficiency but also increases inlet noise at conditions close to surge. Spectral analysis shows that this increase is mainly produced in the form of a medium frequency broadband noise usually known as 'whoosh' in the literature. On the other hand, effect on the outlet is less noticeable. Measurements of the recirculated backflow distortion in terms of circumferential skewness show good correlation with whoosh noise increase, indicating that flow distortion caused by tighter elbows at marginal surge conditions facilitates the transmission of whoosh noise oscillations to the inlet duct, worsening the acoustic behaviour of the system.
\end{abstract}

Keywords: Automotive, Acoustics, Optimization, Turbomachinery, Internal Combustion Engine, NVH

\section{Introduction}

Due to increased pressure to lower harmful emissions and increase fuel efficiency, turbocharging has become commonplace in internal combustion engines from all market segments. Previously restricted to higher performance vehicles, nowadays even smaller automotive engines are equipped with these systems. Moreover, in these applications it is typical that the turbocharger compressor operates at points closer to its deep surge limit, where partially stalled conditions are already in place $[1,2]$.

Different geometric variations of the inlet line near the impeller have been proposed [3-6] in order to mitigate the negative effect of the flow instabilities due to the partial stall and enlarge the air mass flow rate that can be used without risking deep surge conditions.

While studies mainly aimed at obtaining a more stable flow to delay deep surge onset and to keep compressor thermodynamic efficiency even at low air mass flow conditions, the impact of these geometric solutions in the generated and transmitted compressor noise is not often considered.

The acoustic emission of the compressor can be detrimental not only because it contributes to the overall noise created by the engine and thus to the acoustic contamination caused by thermal vehicles, but also because as a very high speed flow machine, its acoustic signature in terms of frequency content

\footnotetext{
*Corresponding author. Email: jorgarti@mot.upv.es
}

differs significantly from that of the reciprocating thermal engine itself.

At operating conditions where the 'blowing' noises of the compressor overpower the deeper, lower frequency combustion noise of the engine, the customer may perceive the engine acoustic response as unpleasant or even faulty, since subjective perception of noise quality is not only tied to overall levels but also frequency content $[7,8]$.

Among the inlet geometries found in automotive turbochargers, it is common [9] to find a $90^{\circ}$ elbow just upstream of the compressor inducer, since tight packaging requirements inside the engine compartment often require the turbocharger to be placed at a side of the engine block.

This particular geometry has been studied in previous research works, both from numerical and experimental standpoints $[10,11]$. While they have demonstrated influence on the surge margin and the efficiency of the compressor [12], literature is scarce on the consequences of $90^{\circ}$ elbow inlets regarding the acoustic output of centrifugal compressors.

In this experimental investigation, three $90^{\circ}$ elbow compressor inlet geometries with different radii are tested in a turbocharger test bench fitted with both piezoelectric pressure sensor arrays to perform in-duct acoustic intensimetry and with thermocouple arrays to characterize the behaviour of the compressor backflow at unstable conditions, thereby allowing the establishment of correlations between the flow field and the acoustic emission. 


\section{Background}

In the last decade, the phenomenon of noise sources in automotive turbochargers has been addressed by several investigations. Gaudé et al. [13] offer a summary of these turbocharger noise sources. They are divided into tonal noise and broadband noise sources. Concerning the latter, a noise is identified with the name of 'hiss' displaying frequency content in the $0-20 \mathrm{kHz}$ range. However, the $0.5-2 \mathrm{kHz}$ interval is chosen to elaborate a compressor sound map wherein the noisiest working points are located at the low mass flow high compression ratio region, near the surge line. That noise origin is attributed to turbulence in the compressor stage.

Evans \& Ward $[1,14]$ found broadband noise similar to previously studied 'whoosh noise' in 9 modern HSDI engines. The most energetic frequency range found was $1.5-3 \mathrm{kHz}$. This noise is noticed in high load acceleration or transient tipin or tip-out manoeuvres. Compressor operating points close to marginal surge line (the locus of zero-slope points of the isospeed lines) appeared to be the noisiest. Turbulence increase associated with this line unstable operation was proposed as the source phenomenon.

Raitor \& Neise [15] mention a 'tip clearance noise', which is a broadband noise found in the $3-5.5 \mathrm{kHz}$ frequency range. In their reported data it can be found as well relevant frequency content in the $1.2-2 \mathrm{kHz}$ and $2-2.8 \mathrm{kHz}$ bands, coherently with previous 'whoosh' reports. The proposed source for those noises are the stalled cells, found as well in axial compressors which display the same acoustic features.

Research by Teng \& Homco [2] shows broadband noise in the $1-2 \mathrm{kHz}$ and $5-7 \mathrm{kHz}$ bands. These authors measured hot backflow at the compressor inducer, caused by the blade stall related to working points close to surge. The reported measurements allowed them to correlate broadband noise to inducer backflow.

In a paper by Figurella et al. [16] broadband noise is measured, called 'whoosh or blow noise', in the $0.8-2 \mathrm{kHz}$ strip and in $4-12 \mathrm{kHz}$ as well. Their soundmaps present an increase of noise in near marginal surge conditions, suggesting again a connection with backflow.

Pai et al. [17] mention 'whoosh' as a broadband noise. Again, near surge working points and transitory tip-in or tipout operations as well as certain steady driving conditions are found to be the noisiest. A mechanism is proposed where acoustic radial mode resonance amplifies frequency content in the $3-4 \mathrm{kHz}$ band.

As a summary, the majority of researchers found broadband noise near the $1-3 \mathrm{kHz}$ band, and attributed it to near surge operation and blade stall related hot backflow. Concerning the inlet geometry, references can be found where different devices are analysed in terms of their influence on compressor performance and stable operation map.

The work by Serrano et al. [4] investigated the effect of different $90^{\circ}$ elbows on inlet flow uniformity and pressure drop. They found a high uniformity index to be desirable, and that although beneficial to surge margin, pressure drop should be avoided in order to preserve an acceptable choke limit. The optimum geometry had the highest curvature radius, and two guiding vanes. Noise response was not studied.

CFD and experimental study was carried out by Li et al. [10] regarding two $90^{\circ}$ elbows with different lengths. Choke limit reduction and efficiency losses were found in comparison with straight duct. Furthermore, unsteady flow at the diffuser was caused by elbow-induced inlet flow distortion. The interaction between such flow and the volute tongue affected the acoustic response by varying the amplitude of the Blade Passing Frequency (BPF) harmonics. Although these spectral pressure content measurements contributed in describing the noise response of the devices, the acoustic issue was not discussed in this paper.

Wang et al. [11] carried out the study of the same geometries, but in this case deeper correlations between the spectral pressure content at certain spots and the compressor performance were drawn. For instance, blade tip spectrum at midchord revealed the influence of intensity and core position of the inlet pipe-induced flow distortion on the blade loading oscillation. This distortion impacted the amplitude distribution of BPF tones at the diffuser, and compressor stage performance as well. Again, the discussion is focused on the machine performance, and the acoustic behaviour is not considered.

Recently, Zhao et al. [12] investigated the effect of orientation angle between a $90^{\circ}$ elbow and the volute tongue. Superposition of inlet pipe flow distortion and high pressure region induced by volute tongue, was found to cause channel blockage and shift choke limit towards lower mass flow rate, thereby reducing stable operating range.

Hou et al. [18] studied two consecutive $90^{\circ}$ elbows with different assembling angles. These ducts generate swirl distortion in the shape of bulk-like and twin counter-rotating vortices, which cause pressure and efficiency losses. The authors found the intensity of those losses to be strongly related to the relative rotating directions of vortices and rotor. In these recent papers the acoustic response was not studied either.

One of the few instances found in recent bibliography of acoustic-focused inlet duct study is a paper by Karim et al. [19]. CFD simulations and experimental measurements were performed on several combinations of swirl vanes and an inlet groove, to test their effect on $4-12 \mathrm{kHz}$ broadband 'whoosh' noise reduction. The combination of swirl vanes and inlet step yielded the best performance in terms of noise. However, the studied device is a straight duct which usually cannot be directly implemented in passenger cars due to the aforementioned packaging restrictions in the engine compartment.

Similarly, Galindo et al. [20] studied a tapered duct, a convergent-divergent nozzle, a convergent one and a baseline straight duct inlet. They found the three new geometries to improve surge margin, efficiency at the surge limit, and sound intensity compared to the baseline duct. Improvements achieved by the nozzle where bigger than those of the tapered duct, being the downside a poorer behaviour in the high mass flow region. As with Karim et al. [19], elbows were not considered in this investigation. 


\section{Experimental setup}

\subsection{Facility \& instrumentation}

To assess the influence of the elbow radius on the recirculating backflow and the acoustic emission of the turbocharger, advantage was taken of an existing test rig built for the implementation and validation of characterization methodologies with a straight duct configuration [21].

In this facility, flow was provided to the turbocharger system installed inside the anechoic chamber from a nearby screw compressor. Combined with a throttle valve at the turbocompressor outlet, air mass flow and pressure ratio were modified in order to test different working conditions across the compressor map.

\subsubsection{Backflow temperature field}

Following the methodology established in [21], the turbocharger inlet was instrumented with two (circumferential

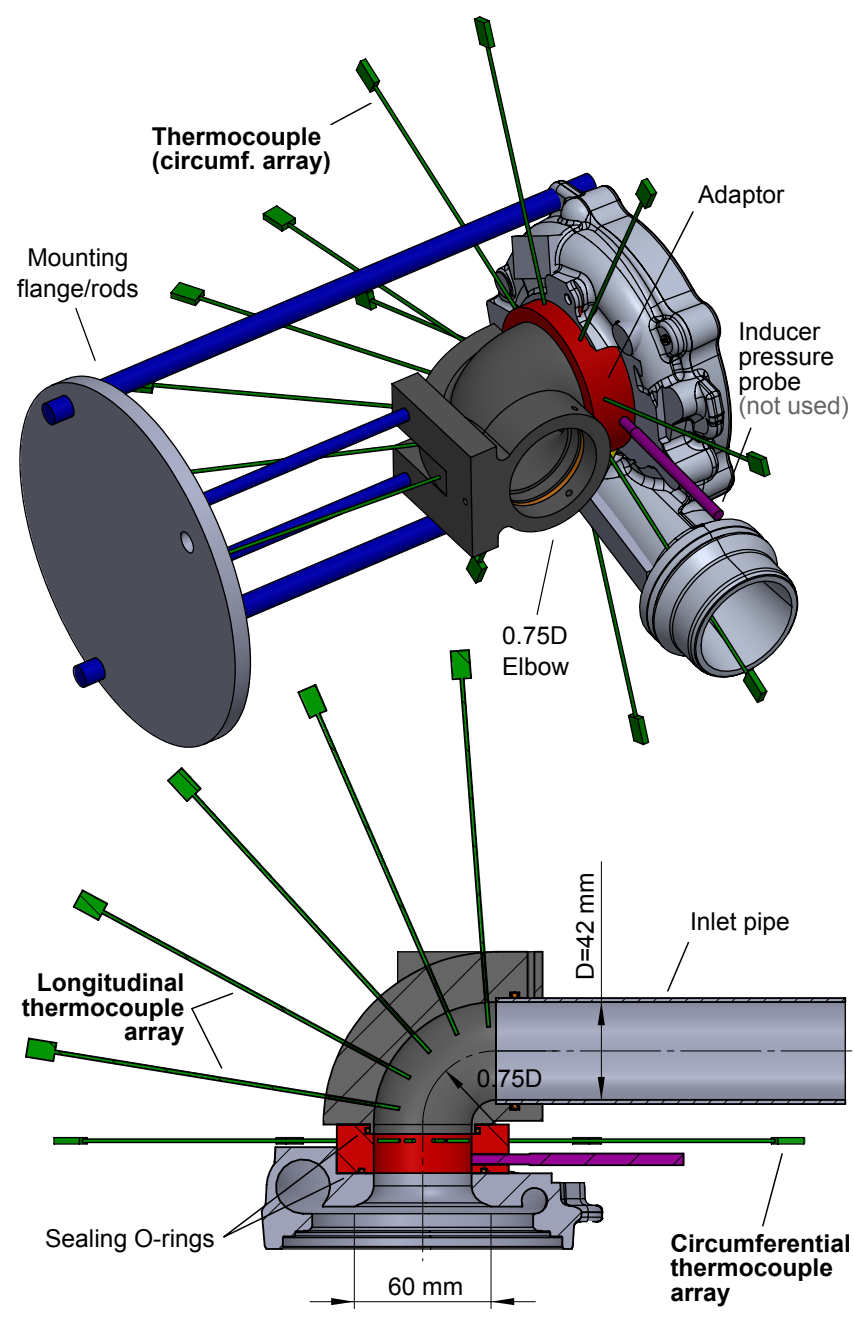

Figure 1: CAD model of the compressor assembly featuring the $0.75 \mathrm{D}$ elbow inlet attached to the common adaptor, including the pressure and temperature instrumentation, the mounting system and a section view of the assembly.

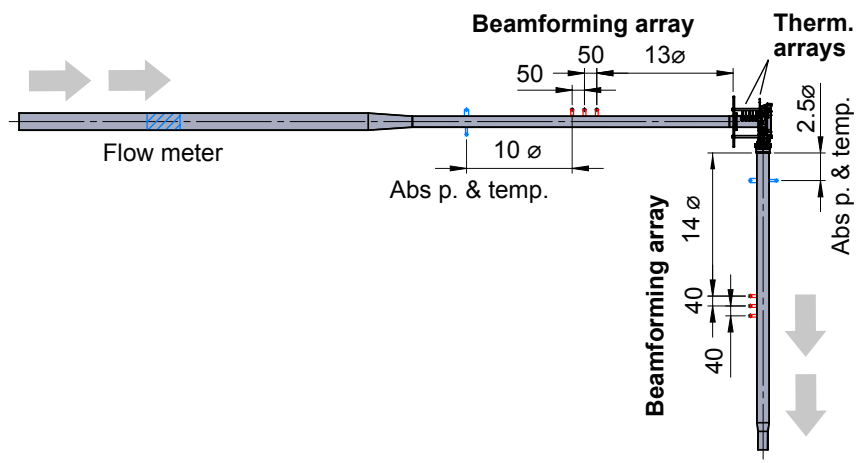

Figure 2: Drawing of the instrumentation for the reference straight inlet configuration, showing the placement of the beamforming and thermocouple arrays along with auxiliary sensors in the ducts. Array separations are given in $\mathrm{mm}$.

and longitudinal) thermocouple arrays, taking advantage of the high temperature of the reversed flow at partially stalled conditions in order to characterize its distribution along the inlet. Fig. 1 shows the assembly of the tighter elbow variant to the compressor housing, featuring the two arrays.

Temperature data from these arrays allowed the estimation of the backflow angular distortion, quantified by the standard deviation of the circumferential array readings, and the upstream extension of the backflow, by analysing the longitudinal temperature profile along the inlet.

In order to facilitate the comparison with the previously published straight duct data, the longitudinal thermocouple array separation was arranged by following the same protrusion ( $1 / 4$ inlet diameters) and separation ( $1 / 3$ diameters) criteria.

The longitudinal array was installed in the outer diameter of the elbows, since for the tighter one it was the only possibility in order to fulfil these criteria. The section view of the bottom drawing of Fig. 1 shows the design of this elbow variant featuring the thermocouple array along its outer radius and the flush mounting with the inlet, preventing any discontinuities that may alter the flow.

\subsubsection{Acoustic intensimetry}

On the other hand, the sound generation of the turbocharger was again assessed through the in-duct acoustic beamforming intensimetry method described by Torregrosa et al. [22]. Beamforming piezoelectric sensor arrays were installed in both inlet and outlet ducts of the turbocompressor at the same distances that in the baseline straight duct case, shown as a reference in Fig. 2. In short, the objective of the beamforming procedure is to decompose the total pressure field $p$ into forward (or downstream) $p^{+}$and backward (or upstream) $p^{-}$pressure waves.

This is achieved by assigning different weights to the data recorded by the three consecutive sensors, thus mathematically favouring the reception of pressure components traversing the array in the $90^{\circ}$ arrival direction and then doing so in the inverse direction. While several strategies can be used to determine these weights, in this case the Linearly Con- 
strained Minimum Variance (LCMV) algorithm [23] is used. Then, sound intensity can be derived following Morfey's [24] expression for a duct with mean flow as:

$$
I(f)=\frac{1}{\rho a}\left(\left|\mathbf{X}^{+}\right|^{2}(1+M)^{2}-\left|\mathbf{X}^{-}\right|^{2}(1-M)^{2}\right)
$$

In this expression $\mathbf{X}^{+}$and $\mathbf{X}^{-}$are the complex spectra of the forward and backward pressure waves $p^{+}$and $p^{-}, \rho$ is the mean density, $a$ the mean sound speed, and $M$ the mean Mach number of the flow. In order to compute the Sound Intensity Level (SIL) in decibels at a certain frequency band $\left(f_{1}, f_{2}\right)$, the following expression can be used, taking into account the usual reference sound intensity of $10^{-12} \mathrm{~W} / \mathrm{m}^{2}$ :

$$
\operatorname{SIL}_{\mathrm{dB}}=10 \log _{10}\left(10^{12} \sum_{f=f_{1}}^{f_{2}} I(f)\right)
$$

\subsubsection{Data acquisition}

In order to keep the best synchronization possible and facilitate postprocessing, data from both beamforming arrays and both longitudinal and azimuthal thermocouple arrays was captured simultaneously with a Yokogawa DL850V digital oscilloscope, which features measurement modules for direct reading of thermocouples along with voltage channels. Voltage channels reading pressure sensors were sampled during $1 \mathrm{~s}$ at $100 \mathrm{kHz}$, allowing an usable frequency range of 50 $\mathrm{kHz}$ for the acoustic spectra characterization.

Hardware clock requirements of the oscilloscope in this configuration dictated that the data from the thermocouples to be sampled at $5 \mathrm{kHz}$, which far exceeds the slow time response of the thermocouples (type $\mathrm{K}$ with $1.5 \mathrm{~mm}$ tips). In any case, since thermocouples readings were to be averaged for the procedure [21], this only represented the inconvenience of slightly higher file size, which was outweighed by the advantage of gathering all scientific data through a single instrument with a common timebase.

Auxiliary pressure and temperature sensors that are required to keep the operating point of the compressor and the auxiliary systems (oil, water, pumps, valves, etc.) but are not directly used in the scientific measurements were captured by an in-house system with a sampling rate of $1 \mathrm{~Hz}$ during 10 s measurements and then averaged. This system also shows real-time traces of the operating values, allowing the operator to ensure that the turbocharger is stabilized in a steady state condition before triggering the capture of each data point.

In Fig. 2 the flow meter and the piezoresistive absolute pressure sensors and thermocouples used to characterize the total flow variables that define the operating point of the compressor are depicted for the reference straight case. The same instrumented ducts were kept for the elbow measurements.

Regarding the uncertainty of the measurements, the piezoelectric pressure sensors used in the acoustic beamforming array are accurate up to $\pm 0.7 \%$. A detailed investigation regarding the uncertainty analysis of different kinds of beamforming algorithms, including the LCMV strategy followed in this investigation can be found in the work of Piñero at al. [25] where it is shown that for an appropriate level of signal-tonoise ratio SNR $>40 \mathrm{~dB}$ (which is greatly exceeded in the turbocharger case), estimation errors of the beamforming procedures are in the order of $1 \mathrm{~dB}$.

As for the thermocouples, uncertainty of the improved type $\mathrm{K}$ class (also referred to as special error class, class 1 , etc. by the different manufacturers) used in this investigation is reported at $1^{\circ} \mathrm{C}$. As in the case with the beamforming procedure, these levels of uncertainty ensured in the previous investigation [21] that the reported rise in temperature field distortion and whoosh noise boost at unstable conditions near surge is well characterized, which was confirmed by the good correlation found between the two phenomena, measured by independent means.

\subsection{Elbow geometry designs}

Requirements for the design of the elbow inlets were such that they could be easily installed into the described existing setup, coupling to the adaptor piece and existent measurement pipe featuring the in-duct intensity sensor array.

In addition, they needed to house the available linear thermocouple arrays in order to measure the high temperature reversed flow (it should be noted that the circumferential thermocouple array was installed into the adaptor piece) approximately keeping the distance between tips and distance from the wall that were selected for the straight pipe study.

Finally, to assess the influence of the flow distortion, the three selected variants were as follows: a very tight elbow with a central radius of 0.75 compressor inlet diameters (marked as D in Fig. 1), a medium elbow with 1D of radius and finally an elbow with a central radius of $1.5 \mathrm{D}$ that would provide a smoother transition and less distorted flow. The three prototypes are shown in Fig. 3.

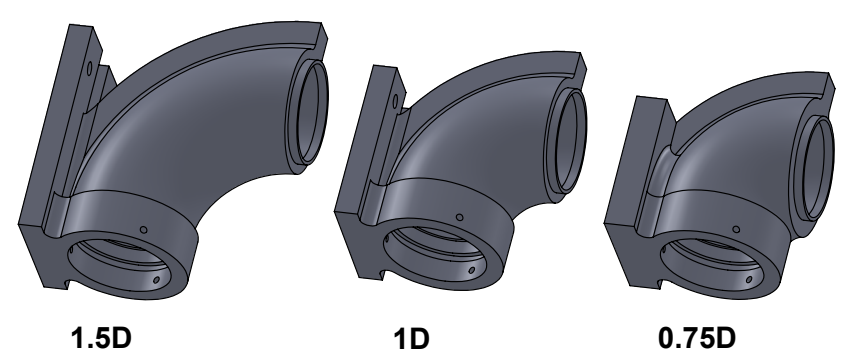

Figure 3: $C A D$ models of the compressor elbow inlets of different radius (decreasing in radius from left to right) that were used to create the $3 D$-printed prototypes.

To fulfil these design requirements, a decision was made to manufacture the elbow prototypes using 3D printing instead of traditional workshop means. This way, the guiding slots for the thermocouples and retention screws could be directly embedded in the piece, along with the slots for the sealing O-rings.

This design was implemented in a parametric CAD model to easily modify the central radius. Also, a locking system was put into place so the elbow could be affixed tightly to 
the compressor adaptor using a flange and a set of threaded rods, similarly to the procedure performed for the straight duct characterization.

It should be noted that the elbow prototypes were expected to withstand the high temperature backflow coming from the compressor in unstable operating conditions close to deep surge, without undertaking deformations that would have misaligned the thermocouple array.

Taking into account these concerns, it was decided to manufacture the elbow prototypes using an aluminium-filled polyamide through a process known as Selective Laser Sintering (SLS), a technique in which a laser beam is focused onto the surface of a tray containing the powdered material, binding it together in layers to create the desired shape.

Specifications of the selected material can be found in table 1 . Of special relevance is the heat deflection temperature, defined as the temperature at which a sample, under a defined load of $1.82 \mathrm{MPa}$, will start to deflect at least $0.25 \mathrm{~mm}$. Since in this case the inlet line is approximately at ambient pressure, the prototypes performed successfully, highlighting the usefulness of this technique for compressor inlet research.

After SLS manufacturing, the prototype elbows were instrumented with the thermocouple arrays and fitted into the turbocharger assembly, which was setup in the aforementioned anechoic test rig. As the narrow holes for the thermocouple alignment became filled with residual dust during the sintering process, and had to be finished with a manual drill. This presented no problem as the chosen material is easily mechanized.

In Fig. 1 it can be seen that the previously used [21] adaptor piece housing the circumferential temperature array, a miniature pressure probe and sealing O-rings was kept in place, and that the holes in the plane surface added to the elbow were used to align the elbow parallel to the outlet, tightening it using the threaded rods depicted in blue (one is removed for better visibility).

\section{Results and discussion}

During the experimental campaign, operating conditions were selected to mirror those used in the previously reported straight inlet pipe characterization [21], in order to allow

Table 1: Specifications of the aluminium-filled polyamide used for the laser-sintering of the elbow inlet prototypes.

\begin{tabular}{llc}
\hline Property & Unit & Value \\
\hline Tensile Modulus & $\mathrm{MPa}$ & $3800 \pm 150$ \\
Tensile Strength & $\mathrm{MPa}$ & $48 \pm 3$ \\
Elongation at Break & $\%$ & $3.5 \pm 1$ \\
Flexural Modulus & $\mathrm{N} / \mathrm{mm}^{2}$ & $3600 \pm 150$ \\
Charpy - Impact strength & $\mathrm{MPa}$ & $29 \pm 2$ \\
Charpy - Notched Impact Strength & $\mathrm{MPa}$ & $4.6 \pm 0.3$ \\
Shore D/ A-hardness & - & $\mathrm{D} 76 \pm 2$ \\
Heat Deflection Temp (ASTM D648 1.82MPa) & ${ }^{\circ} \mathrm{C}$ & 130 \\
Vicat Softening Temperature B/50 & ${ }^{\circ} \mathrm{C}$ & 169 \\
Density & $\mathrm{g} / \mathrm{cm}^{3}$ & $1.36 \pm 0.05$ \\
\hline
\end{tabular}

the reconstruction of interpolated maps that are as close as possible to the baseline map, encompassing the left hand side of the compressor map where speed line slopes are small and partial or mild surge is found, being also the region of interest for automotive applications.

Fig. 4 shows the operating conditions of each measured data point, for both the straight inlet pipe used as reference, and for the three described elbow prototypes. It can be seen that repeatability was achieved in most conditions up to a few $\mathrm{g} / \mathrm{s}$ of air mass flow and 100 corrected rpm.

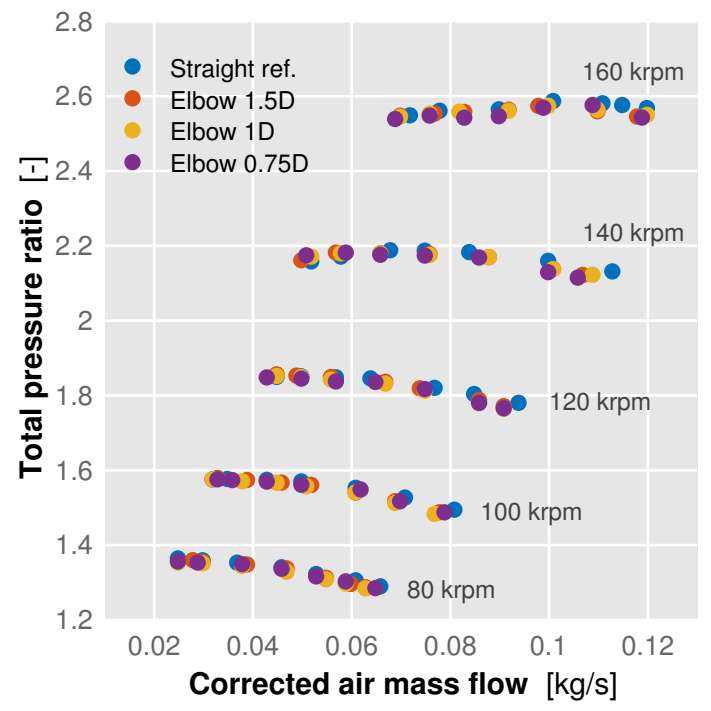

Figure 4: Operating conditions at which data was recorded during the inlet flow characterization tests with a straight duct (as reference) and during the parametric elbow testing. These same data points will be used in all map figures.

The most adverse conditions were those of maximum allowable flow, especially at higher pressure ratios where the flow distortion and pressure loss caused by the elbows made difficult to achieve exactly the same conditions.

\subsection{Sound intensity}

Using the in-duct piezoelectric sensor arrays, sound intensity level was computed through the wave decomposition method for each operating condition. In order to focus into the previously described whoosh noise problem, sound intensity level was averaged between 0.7 and $3 \mathrm{kHz}$ following Eq. 2 and then the interpolating procedure described in [22] was used in order to reconstruct noise maps for each geometry.

To highlight the differences between the reference straight duct noise map and the result obtained for each elbow prototype, and taking into account the aforementioned slight differences between operating conditions at higher air mass flow settings, difference maps were produced by subtracting the reference levels to each one of the newly obtained elbow maps.

It should be remarked that this subtraction procedure was not performed between the raw levels at each measurement setting but between each interpolated data point, and only 


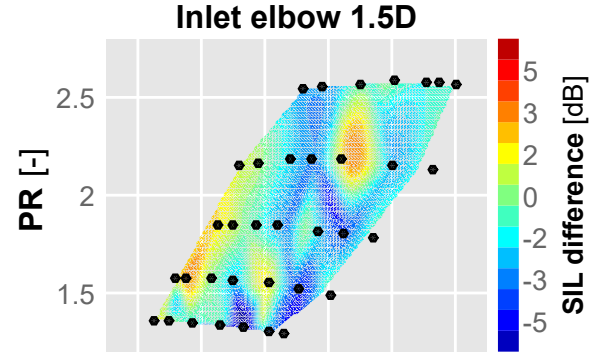

$0.020 .04 \quad 0.060 .08 \quad 0.1 \quad 0.12$ AMF $[\mathrm{kg} / \mathrm{s}]$

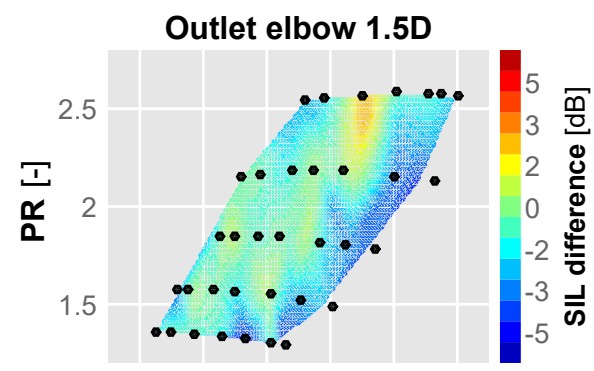

$0.020 .04 \quad 0.060 .08 \quad 0.1 \quad 0.12$ AMF $[\mathrm{kg} / \mathrm{s}]$

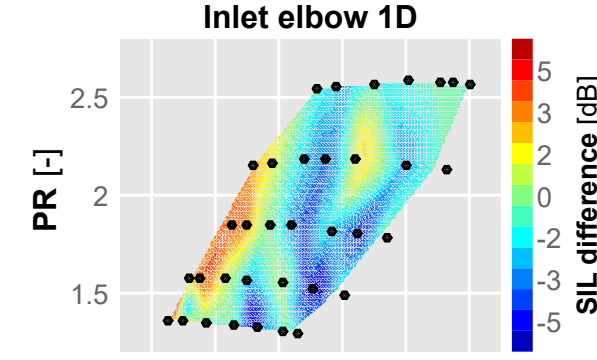

$0.020 .040 .060 .08 \quad 0.1 \quad 0.12$ AMF $[\mathrm{kg} / \mathrm{s}]$

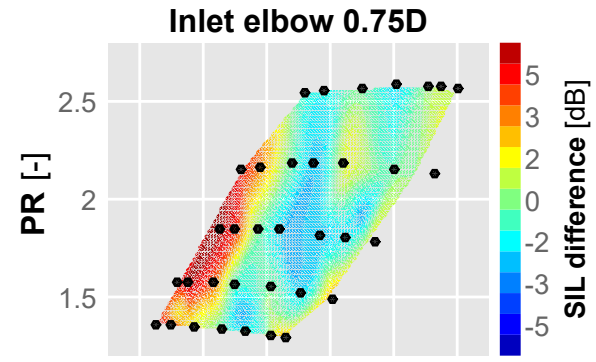

$0.020 .040 .060 .08 \quad 0.1 \quad 0.12$ AMF $[\mathrm{kg} / \mathrm{s}]$

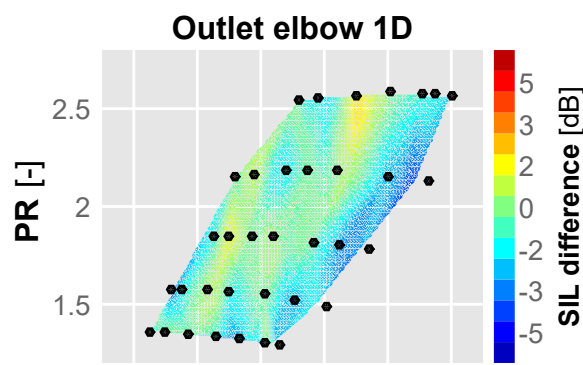

$0.020 .040 .060 .08 \quad 0.1 \quad 0.12$ AMF $[\mathrm{kg} / \mathrm{s}]$

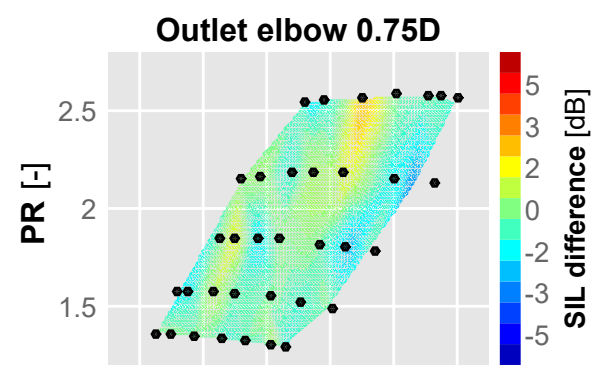

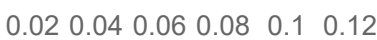
AMF $[\mathrm{kg} / \mathrm{s}]$

Figure 5: Difference map of inlet (top) and outlet (bottom) in-duct sound intensity levels for each of the selected inlet elbows compared against the reference straight duct. From left to right: $1.5 D, 1 D \& 0.75 D$.

where such interpolated data existed in both noise maps (elbow and reference), in order to avoid erroneous differences due to the different operating conditions.

The results of this procedure can be seen in Fig. 5, where the difference maps for the three elbow configurations regarding inlet and outlet in-duct intensity are presented. Colour scale has been kept constant in order to allow a meaningful comparison between each map.

It can be seen in the maps, examining for instance some of the highest mass flow rate points, that some information is missing around the black dot that indicates the original measurement point before interpolation. This missing data comes from slight differences in measurements between the two geometries being compared, as the subtraction process is only performed where data exists for both of them.

As for the evolution of level difference with the variation of the elbow radius, the figure shows how in the case of the inlet sound intensity differences appear to be higher than in the case of the outlet. This increase is especially evident at lower flow conditions and medium-low shaft speeds, reaching up to $6 \mathrm{~dB}$ in level increase.

There also appears to be a particular point with an small increase in level for both inlet and outlet ducts, at medium air mass flow and medium to high shaft speeds, its relevance diminishing along with the reduction of the elbow radius.

Finally, some operating conditions in both the inlet and outlet present reduced noise output in the selected frequency band, especially at medium-higher mass flows and lower shaft speeds. As with the case of the increases, these reductions are more significant in the inlet duct, whereas positive and
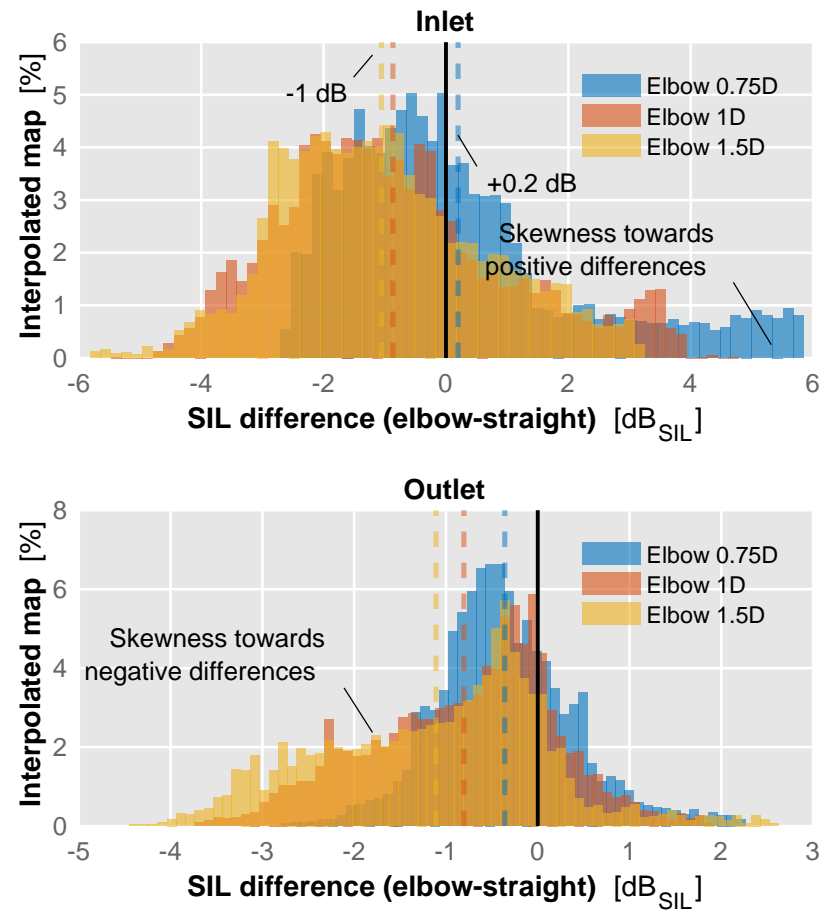

Figure 6: Histograms of inlet (top) and outlet (bottom) sound intensity level differences between each one of the tested elbow variants and the reference straight inlet pipe. $Y$ axis indicates the percentage of the interpolated compressor map at which each difference level occurred. A dashed line indicates the average of each configuration. 

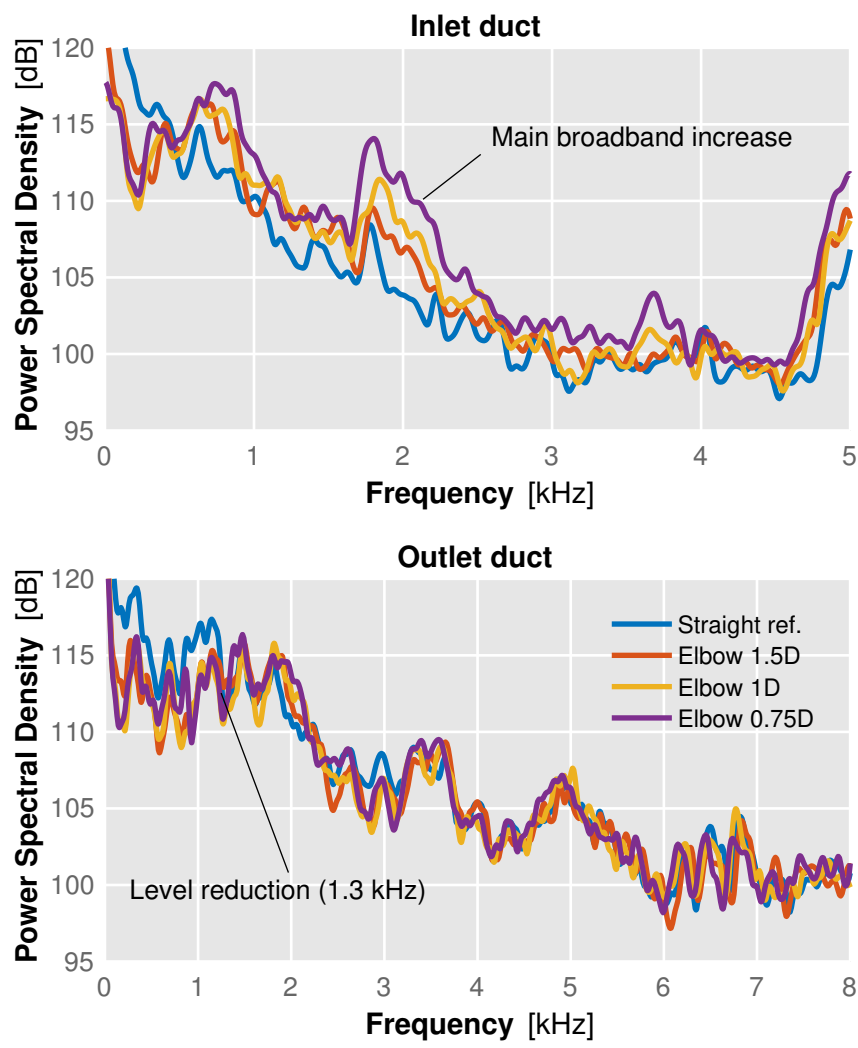

Figure 7: Comparison of plane wave range spectra from both inlet and outlet, highlighting the differences between the straight reference duct and each elbow configuration at 120 $\mathrm{krpm}$ and $45 \mathrm{~g} / \mathrm{s}$.

negative variations are of smaller magnitude in the outlet duct.

In order to better quantify the SIL differences that these maps reveal, histograms that represent the distribution of the differences have been computed and presented in Fig. 6 for both inlet (top) and outlet (bottom) sound intensity results.

It can be seen in the figure how the different elbow configurations affect the distribution of the intensity level differences. In the case of the inlet, the less narrow elbow (1.5D) and the medium elbow (1D) present a similar, almost symmetric distribution with a mean around $-1 \mathrm{~dB}$.

The distribution of the most narrow elbow (0.75D) appears to feature a marked skewness towards positive differences of up to $6 \mathrm{~dB}$ that correspond with the low flow, mediumlow speed region identified in the aforementioned intensity maps. These points shift the mean up to approximately 0.2 $\mathrm{dB}$ of increased level.

Regarding the outlet difference distributions, the situation appears to reverse, with the narrower elbow now featuring the most symmetric distribution and the other two variations presenting a marked skewness towards intensity reduction values, again up to a mean of around $2 \mathrm{~dB}$ in the most favourable case (1.5D elbow).

Focusing now into the detailed aspects of the sound level variation, it is possible to compare the pressure spectra in different points to assess the frequency distribution of the level variation. For instance in Fig. 7 the PSD in the plane wave region recorded by inlet and outlet piezoelectric sensors is shown for the different inlet geometries at an operating condition where differences were high (120 krpm, $45 \mathrm{~g} / \mathrm{s}$ ).

In the case of the outlet duct, spectra are very similar up to a cut-off point at approximately $1.3 \mathrm{kHz}$, below which the straight duct presents higher levels than all of the elbows.

On the other hand, spectra on the plane wave region of the inlet duct shows higher differences, consistent with the data already presented in the maps and histograms.

It is interesting to note however that this increase is not homogeneous in frequency; it can be seen how the small peak at $1.7 \mathrm{kHz}$ present in the straight duct gradually increases with elbow radius reduction, up to a clearly visible broadband between 1.5 and $2.5 \mathrm{kHz}$.

These are flanked by another two smaller broadband elevations at 0.75 and $3.6 \mathrm{kHz}$ that are also visible in the case of the outlet spectra, and were also very clearly present in the pressure spectra recorded by the diffuser pressure probe, shown for instance in data from [21].

It should be noted as well that, while levels for the detected broadband in the inlet show an increase up to $5 \mathrm{~dB}$, at these frequencies all of the outlet spectra plotted in the lower graph remain at the same levels, indicating that while changes in the inlet configuration seem to boost the upstream transmission of the so-called whoosh broadband noise, no influence on the outlet duct spectra is detected at these frequencies.

\subsection{Temperature field}

Besides the described differences in terms of acoustic intensity produced by the different inlet configurations, it is possible to follow a similar processing procedure in order to assess the differences in terms of temperatures in the inlet section that are related to the hot reversed flow coming from after the compressor impeller.

Following the previous experimental campaign, data from the two thermocouple arrays (longitudinal and circumferential) was processed in order to compile maps of the backflow length and flow temperature skewness, respectively.

These maps were then compared with the original reference map measured with a straight inlet duct, and difference maps were produced for each elbow variant. The resulting difference (elbow minus straight) maps can be seen in Fig. 8 .

The top row of this figure shows the difference in circumferential standard deviation, whereas the bottom row depicts the difference in estimated backflow length. In both cases the colour has been kept the same in the three elbows to provide a suitable comparison.

Regarding the circumferential SD (top row) it can be seen that for the three cases a similar patters appears. An increase in temperature skewness is consistently detected at the lower air mass flow settings, especially at medium shaft speed operating conditions.

At higher air mass flow settings however, a slight reduction in flow temperature skewness is apparent, being much more 


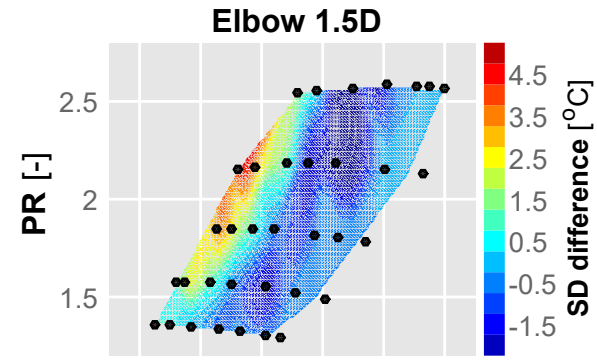

$0.020 .040 .060 .08 \quad 0.1 \quad 0.12$ AMF $[\mathrm{kg} / \mathrm{s}]$

Elbow 1.5D

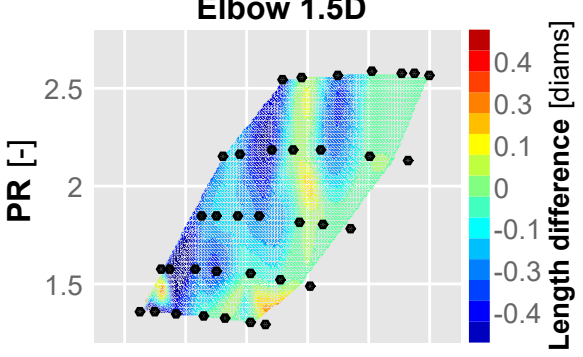

$0.020 .040 .060 .08 \quad 0.1 \quad 0.12$ AMF $[\mathrm{kg} / \mathrm{s}]$

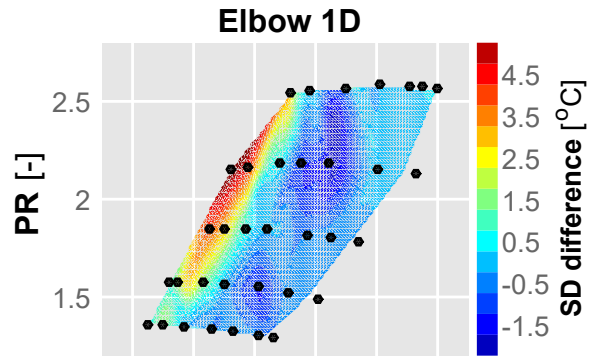

$0.020 .040 .060 .08 \quad 0.1 \quad 0.12$ AMF $[\mathrm{kg} / \mathrm{s}]$

Elbow 1D

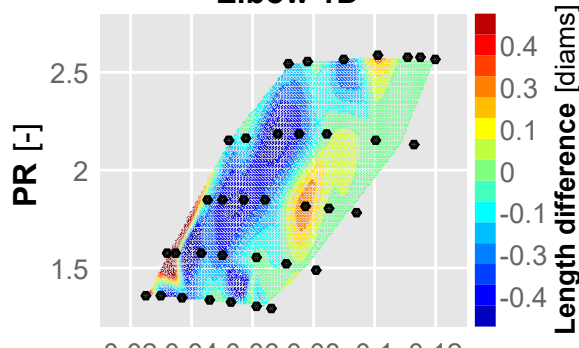

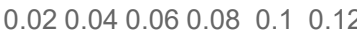
AMF $[\mathrm{kg} / \mathrm{s}]$

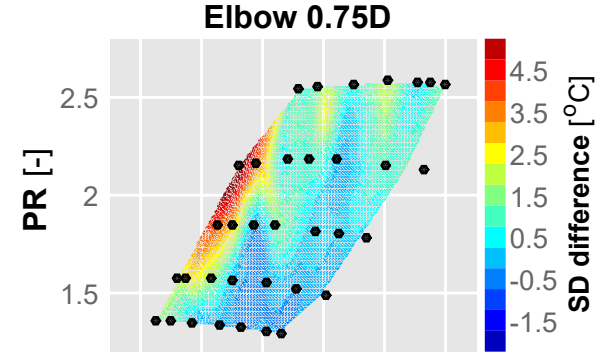

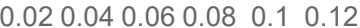
AMF $[\mathrm{kg} / \mathrm{s}]$

Elbow 0.75D

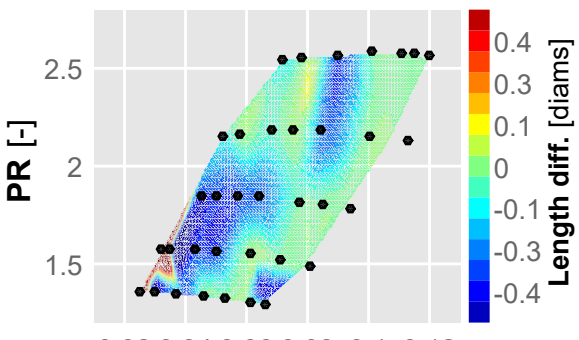

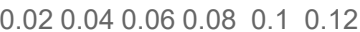
AMF $[\mathrm{kg} / \mathrm{s}]$

Figure 8: Difference maps of temperature circumferential standard deviation (top row) and backflow length (bottom row) for each of the selected inlet elbows compared against the reference straight duct.

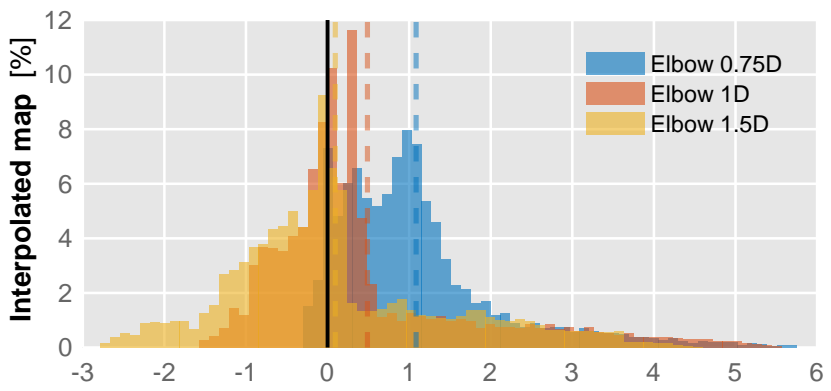

Circumf. temp. SD difference (elbow-straight) $\left[{ }^{\circ} \mathrm{C}\right]$

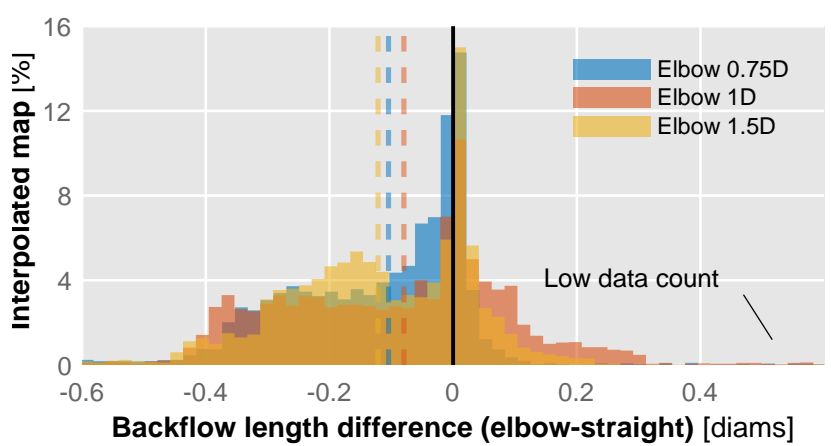

Figure 9: Histograms of temperature $S D$ (top) and backflow length (bottom) differences between each one of the tested elbow variants and the reference straight inlet pipe. $Y$ axis indicates the percentage of the interpolated compressor map at which each difference level occurred. A dashed line indicates the average of each configuration. pronounced in the less narrow elbow configuration (1.5D, top left map). This reduction area spans approximately the same conditions for the $1 \mathrm{D}$ elbow, but the magnitude of the temperature skewness difference is lower than in the previous case, becoming nearly zero in the case of the most reduced elbow variant $(0.75 \mathrm{D})$.

Regarding the estimated length along the exterior edge of the elbow (where the thermocouple array was installed), the bottom row of Fig. 8 shows this length mapped for the three elbow configurations.

In this case, differences range from minus half a diameter up to plus a diameter when compared with the reference straight pipe. The patterns of these differences along the compressor map appears to be similar for the three geometric variations: a slight reduction at lower mass flow conditions and a slight increase at higher mass flow conditions for all shaft speeds.

Exceptions to this pattern are some of the lowest air mass flow data points at 100 and $120 \mathrm{krpm}$ for the two narrower elbows (0.75D and 1D), where the backflow length appears to feature a large increase. However it is possible that those are spurious results from the interpolation procedure, since the increase region is very small.

As in the case of sound intensity level differences, it is possible to combine these maps in the form of histograms in order to better quantify the distribution of these differences. These histograms can be seen plotted in Fig. 9.

In the case of the circumferential skewness (top plot), distributions shown in this figure appear similar in shape, but shifted in mean value. In all three cases the right tail of the 

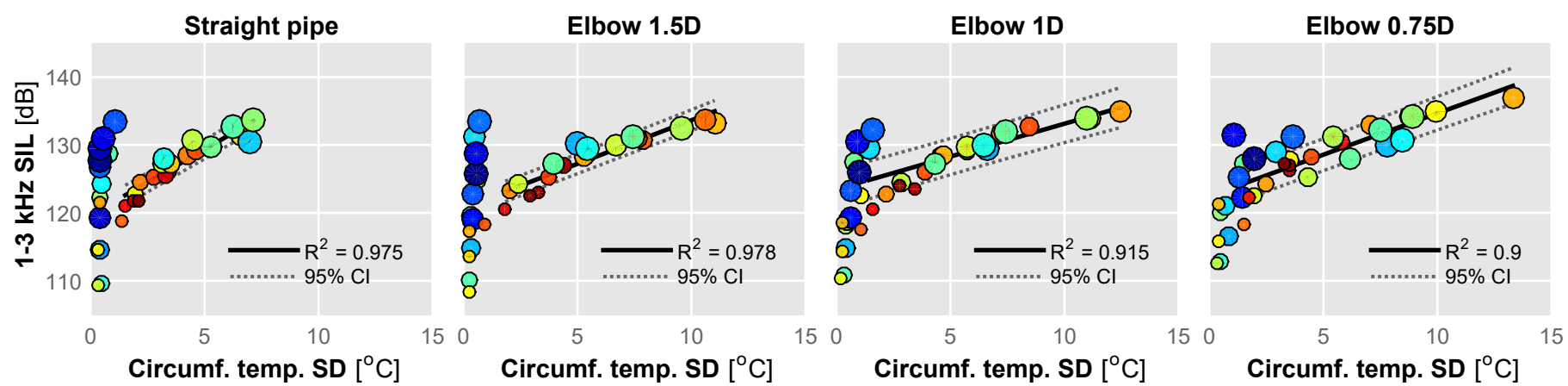

Figure 10: Correlations between standard deviation at the circumferential thermocouple array and inlet sound intensity level in the 1 $3 \mathrm{kHz}$ band for the straight pipe and the three elbow inlets. Size indicates shaft speed from low to high and colour indicates mass flow from higher (blues) to lower (reds).

distribution is longer but less populated, indicating that the difference increase happens abruptly in a small region of the compressor map.

On the contrary, the left tails are more populated but shorter, meaning that more regions of the compressor map feature points with slightly lower differences than the average. These averages reach up to $1^{\circ} \mathrm{C}$ in the case of the most narrow elbow configuration $(0.75 \mathrm{D})$, while the average is almost zero for the case of the less narrow elbow (1.5D).

In the case of the backflow length, the aforementioned low count points are best shown when the data is put into histogram form as seen in the bottom plot of Fig. 9. Indeed it can be seen how the three backflow length difference distributions are quite similar in shape and values.

The three distributions feature approximately the same mean, around 0.1 diameters of backflow length reduction from the straight inlet case. However, the most usual difference (that is, the statistical mode) is practically zero. It must be also considered that the estimation of backflow is only performed along the outer edge of the elbow, while the modified flow pattern could mean that a very non-axisymmetric backflow is propagating upstream.

\subsection{Correlation between SIL and temperature}

In Fig. 10 a correlation between sound intensity level and circumferential temperature skewness has been performed again for the three elbow inlet cases as it was already made in [21] for the case of the straight pipe. This correlation has been included as a reference, keeping the same axis limits of the elbow cases.

It can be seen in this figure how for the straight pipe and the more straight elbow (that with an central radius equal to $1.5 \mathrm{D}$ ), both pictured on the top plots, a clear separation is maintained between higher flow rate conditions (indicated as blue circles) which exhibit higher noise levels even when no skewness is detected, and those of lower flow rate where skewness is well correlated with the increase in noise levels.

In the case of the more narrow elbows, pictured in the bottom plots of the figure, the difference between the higher and lower flow rate cases becomes blurred, as even points of the higher flow rate demonstrate measurable temperature skewness as the elbow radius decreases.

By comparing the four plots of the figure, it can also be seen how the maximum values of temperature skewness increase as the radius of the pipe decreases, with the same trend being followed by the sound intensity level in the selected frequency band, as was clearly seen previously in Fig. 6 where the characteristic 'hump' of whoosh noise becomes more pronounced as the radius of the elbow gets narrower (while a similar influence is not observed in the outlet spectra).

\subsection{Isentropic efficiency}

Once that the relationship between stalled flow recirculation and noise intensity has been discussed, the question may remain as to what is the effect of the modified inlet flow field imposed by the chosen geometries on the efficiency of the compressor.

In order to quantify this influence, the isentropic efficiency $\eta_{s}$ of the compressor can be calculated by dividing the ideal power $\dot{W}_{s}$ required assuming the isentropic compression of an ideal gas, by the actual required power $\dot{W}$ :

$$
\eta_{s}=\frac{\dot{W}_{s}}{\dot{W}}=\frac{\Delta h_{\mathrm{s}}}{\Delta h}=\frac{T_{\mathrm{in}, \mathrm{T}}\left(\Pi_{\mathrm{TT}}^{\frac{\gamma-1}{\gamma}}-1\right)}{T_{\mathrm{out}, \mathrm{T}}-T_{\mathrm{in}, \mathrm{T}}}
$$

Where $T_{\text {in/out, } T}$ represents the total temperature at the inlet or outlet, $\Pi_{\mathrm{TT}}$ is the total-to-total pressure ratio, $\gamma$ is the specific heat ratio of air and 's' denotes an isentropic process.

Inlet and outlet temperatures in this case were measured at the same points where static pressures are recorded by means of piezoresistive transducers, using two pairs of additional thermocouples. Thereby, these temperature readings are not affected by the recirculating hot backflow that could interfere with the calculation.

In Fig. 11 the difference in percentage points (pp) between the straight duct configuration and each of the elbows is plotted. In this case, it is shown how the larger radius elbow improves the efficiency, especially at the lowest air mass flow settings at the bottom left corner of the map. 

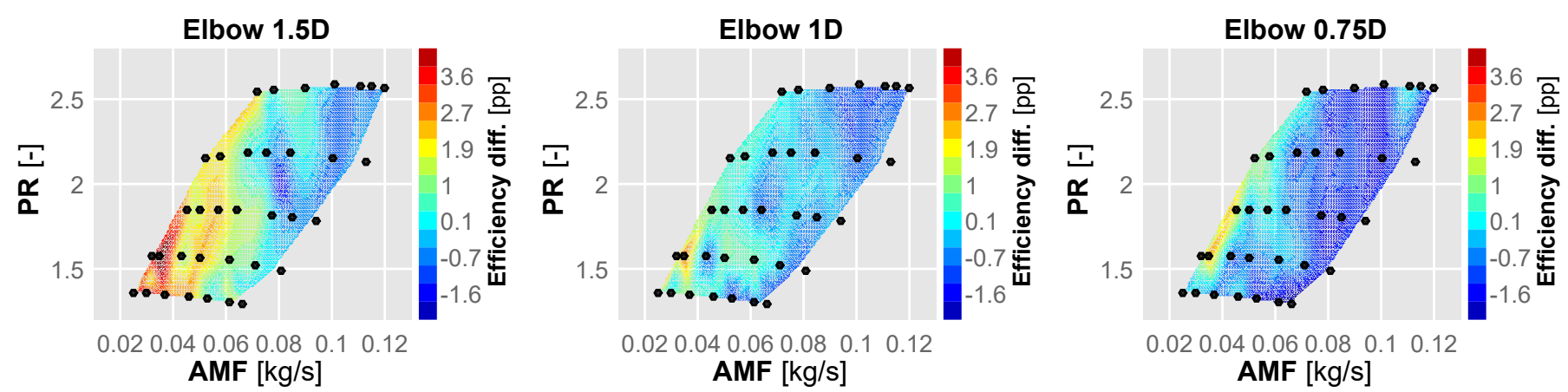

Figure 11: Difference map of isentropic efficiency for each of the selected inlet elbows compared against the reference straight duct. From left to right: $1.5 D, 1 D \& 0.75 D$.

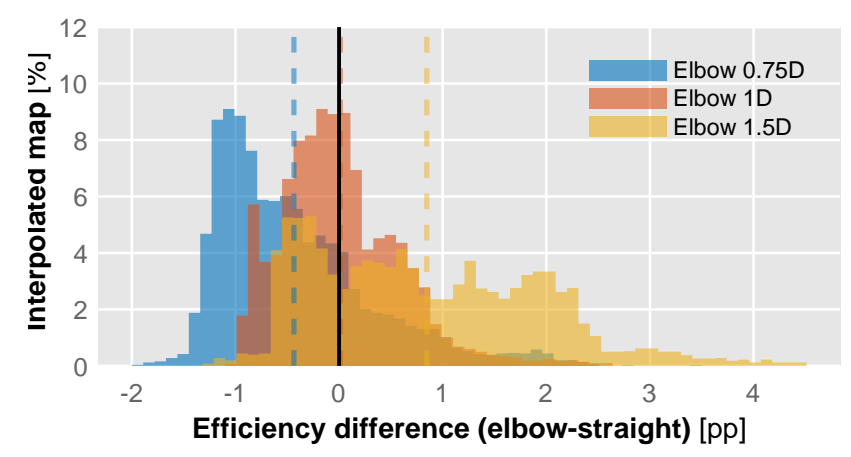

Figure 12: Histogram of isentropic efficiency differences between each one of the tested elbow variants and the reference straight inlet pipe. $Y$ axis indicates the percentage of the interpolated compressor map at which each difference level occurred. A dashed line indicates the average of each configuration.

It is precisely in this region of the map where the simulations of the 'clocking effect' of a $90^{\circ}$ elbow performed by Zhao et al. [12] demonstrated a similar change in efficiency $(\sim 2$ pp) as the angular orientation of their inlet elbow rotated.

On the other hand, efficiency is worsened at the higher mass flows regimes, a tendency which is corroborated as the elbow radius is decreased. While a slight improvement over the reference straight inlet is preserved at the low flow, low speed corner in the 1D and 0.75D inlets, efficiency at the higher mass flow regimes is decreased, especially with the narrower elbow.

This is best quantified by Fig. 12 in which the distributions of efficiency differences in percentage points is plotted in histogram form. It can be seen that the average of the 1D elbow is almost exactly zero, with differences distributed between \pm 1 pp of the straight inlet reference values.

In the case of the narrower $0.75 \mathrm{D}$ elbow, the aforementioned efficiency losses at higher mass flows move the average to -0.5 pp worse efficiency. On the other hand, the less narrow 1.5D elbow features values displaced to the positive half-plane of the chart, demonstrating a +1 pp better efficiency than the original straight inlet.
Whereas the efficiency decrease caused by elbows at higher mass flow can be explained by the flow distortion, which is known to cause loss of efficiency in compressors, it is not entirely clear which mechanism is behind the measured improvement of $2 \mathrm{pp}$ in efficiency between the 1.5D elbow and the straight duct.

An explanation could be offered by the fact that elbows accelerate the flow as the cross-section area is restricted by detachments. This increase in momentum in a particular area could counteract, disrupt or delay the rotating stall mechanism, and thus recover some efficiency lost with the straight duct due to the stalling flow instabilities.

Other flow effect that must be investigated is the swirl induced by the elbow, which according to Kim et al. [26] is still detectable at $10 \mathrm{D}$ from the elbow exit. This swirl could slightly vary the incidence angle [12] and thus assist in keeping the flow correctly attached to the blades.

Finally, although perhaps a second order effect, in centrifugal compressors the presence of the volute tongue causes non-axisymmetric static pressure conditions [27] that affect the flow through the impeller and thus the efficiency [28]; circumferential pressure differences caused by Dean vortices [29] in the elbow could counteract this negative effect.

\section{Summary}

In this investigation, an experimental campaign was carried out where a family of $3 \mathrm{D}$ printed $90^{\circ}$ elbow inlets with different radii were installed on an anechoic test rig and instrumented to measure not only in-duct noise but also the recirculating backflow through its temperature distribution, by means of thermocouple arrays.

Results from this campaign provided insight into the influence of the elbow radius. It was shown that on average, the elbow geometry can slightly reduce in-duct noise levels, especially at the outlet. However, this variation was not uniformly distributed across the compressor map. Higher noise emission was found concentrated on the lowest allowable air mass setting for each shaft speed. This pattern was shared among the three tested elbow configurations, although the 
effect of noise increase at partially stalled condition became more pronounced as the radius became smaller.

Regarding the influence of the inlet elbow variants on the temperature distribution, a similar pattern of circumferential skewness increase towards the lowest allowable flow conditions for each shaft speed was reconstructed. However, reversed flow length appeared similar in all cases, with differences of less than 0.5 inlet diameters. It is in the case of the circumferential skewness of the recirculating backflow where the influence of the elbow geometry became much more apparent.

Correlations of whoosh noise content and temperature skewness indicated an increase of both variables as the elbow radius became narrower. Explanations for these correlated increases could be related to the uneven presentation of the air flow caused by the progressively narrow $90^{\circ}$ elbows. It is known that these vortices create a region of lower pressure [29] that in this case could have facilitated the upstream propagation of reversed flow and thus enhanced the convection of whoosh noise content from the diffuser to the inlet duct, while also increasing the differences in temperature across the different zones.

This fluid-dynamic mechanism offers a reasonable explanation as to why elbow radius demonstrated a clear effect on the whoosh noise content in the inlet spectra shown in Fig. 7 but not in the outlet, and also why this effect is restricted to the lower mass flow rate zones of the compressor map (see Fig. 5 for the effect on sound intensity and Fig. 8 for the effect on temperature skewness) where reversed flow is more prominent, as in this zone instabilities grow while inflow momentum diminishes.

Impact of the $90^{\circ}$ elbow radius on compressor isentropic efficiency was also assessed, in order to enlarge previous studies which considered the influence of the orientation in this parameter. Results evinced that the elbow radius can improve or worsen the efficiency, with this impact being highly dependant on the operating conditions.

As in the previous issue regarding convected whoosh noise, the specific interaction of the flow configuration induced by the elbows with the compressor flow field is the most probable explanation behind the observed performance behaviour. While increased flow distortion due to Dean vortices as the radius tightens is known to generally cause efficiency losses, the swirling motion, together with a the counter-action of the volute tongue perturbation, could help in keeping the flow attached to the blades, thereby improving the efficiency at very low air flow conditions as observed. However, further research on this issue requires the characterization of the flow uniformity and attachment, possibly by using CFD simulations that exceed the experimental scope of this work.

\section{Conclusions}

Arising from the findings summarised above, some conclusions can be drawn from both methodological and phenomenological standpoints. On one hand, the methodology introduced in [21] which aims to characterize the recirculating backflow at partially stalled flow conditions by using the temperature field as a proxy, has evinced good correlation with acoustic beamforming measurements even when facing the flow features of the narrowest elbow, demonstrating its potential to quantify the distortion of the inlet flow field in a simple and robust way that can be easily implemented in research or industrial applications.

On the other hand, results have demonstrated the influence of inlet elbow radius on the most relevant quality variables of the compressor at different flow and speed regimes. While different flow-dynamic mechanisms responsible for these effects have been discussed in the precious section, the specific boosting of the whoosh broadband correlated with flow and temperature distortion is the most noteworthy finding of this work. This fact confirms the importance of better understanding the interaction between the flow distortion caused by the inlet geometry and the non-axisymmetric instabilities of the compressor itself, since even small design changes can have an impact not only on efficiency or surge margin but also on the noise emission of the system.

\section{Acknowledgements}

The equipment used in this work has been partially supported by the Spanish Ministerio de Economía y Competitividad through grant DPI2015-70464-R and by FEDER project funds 'Dotación de infraestructuras científico técnicas para el Centro Integral de Mejora Energética y Medioambiental de Sistemas de Transporte (CiMeT), (FEDER-ICTS-2012-06)' framed in the operational program of unique scientific and technical infrastructure of the Spanish Ministerio de Economía y Competitividad. F. Roig is partially supported through the Programa de Apoyo para la Investigación y Desarrollo of Universitat Politècnica de València (PAID-01-17).

\section{References}

[1] D. Evans, A. Ward, Minimizing Turbocharger Whoosh Noise for Diesel Powertrains, SAE Technical Paper 2005-01-2485. doi:10.4271/ 2005 - 01- 2485.

[2] C. Teng, S. Homco, Investigation of Compressor Whoosh Noise in Automotive Turbochargers, SAE Int. J. of Passeng. Cars-Mech. Syst. 2 (1) (2009) 1345-1351. doi : 10.4271/2009-01-2053.

[3] J. Galindo, J. R. Serrano, X. Margot, A. Tiseira, N. Schorn, H. Kindl, Potential of flow pre-whirl at the compressor inlet of automotive engine turbochargers to enlarge surge margin and overcome packaging limitations, International journal of heat and fluid flow 28 (3) (2007) 374-387. doi:10.1016/j.ijheatfluidflow.2006.06.002.

[4] J. R. Serrano, X. Margot, A. Tiseira, L. M. García-Cuevas, Optimization of the inlet air line of an automotive turbocharger, International Journal of Engine Research 14 (1) (2013) 92-104. doi:10.1177/ 1468087412449085.

[5] E. Guillou, R. DiMicco, E. Gutmark, A. Mohamed, M. Gancedo, Characterization of a Ported Shroud Compressor using PIV Measurements, Tech. Rep. 2010-01-1225, SAE Technical Paper (2010). doi : 10.4271/ 2010 - 01 - 1225.

[6] H. Tamaki, M. Unno, R. Tanaka, S. Yamaguchi, Y. Ishizu, Enhancement of centrifugal compressor operating range by control of inlet recirculation with inlet fins, Journal of Turbomachinery 138 (10) (2016) 101010. doi: 10.1115/1.4033187. 
[7] C. J. da Silveira Brizon, E. B. Medeiros, Combining subjective and objective assessments to improve acoustic comfort evaluation of motor cars, Applied Acoustics 73 (9) (2012) 913-920. doi : 10.1016/j . apacoust 2012.03.013.

[8] M. Nor, M. Fouladi, H. Nahvi, A. Ariffin, Index for vehicle acoustical comfort inside a passenger car, Applied Acoustics 69 (4) (2008) 343353. doi : 10.1016/j . apacoust. 2006.11.001.

[9] A. K. Vester, S. S. Sattarzadeh, R. Örlü, Combined hot-wire and piv measurements of a swirling turbulent flow at the exit of a $90^{\circ}$ pipe bend, Journal of Visualization 19 (2) (2016) 261-273. doi : 10.1007/ s12650-015- 0310- 1.

[10] D. Li, C. Yang, M. Zhou, Z. Zhu, H. Wang, Numerical and experimental research on different inlet configurations of high speed centrifugal compressor, Science China Technological Sciences 55 (1) (2012) 174 181. doi : $10.1007 /$ s 11431 - 011 - 4635 - 2.

[11] L. Wang, C. Yang, B. Zhao, D. Lao, C. Ma, D. Li, The change of the inlet geometry of a centrifugal compressor stage and its influence on the compressor performance, Journal of Thermal Science 22 (3) (2013) 197-208. doi : 10.1007/s11630-013-0613- 2.

URL http://dx.doi.org/10.1007/s11630-013-0613-2

[12] B. Zhao, H. Sun, L. Wang, M. Song, Impact of inlet distortion on turbocharger compressor stage performance, Applied Thermal Engineeringdoi: 10.1016/j . applthermaleng . 2017.05.181.

[13] G. Gaudé, T. Lefèvre, R. Tanna, K. Jin, T. J. B. McKitterick, S. Armenio, Experimental and computational challenges in the quantification of turbocharger vibro-acoustic sources, in: Proceedings of the 37th International Congress and Exposition on Noise Control Engineering (INTER-NOISE 2008), Vol. 2008, Institute of Noise Control Engineering, 2008, pp. 5754-5767.

URL http://WwW.ingentaconnect.com/content/ince/incecp/ 2008/00002008/00000003/art00009

[14] D. Evans, A. Ward, The reduction of turbocharger whoosh noise, in: Proceedings of the 8 th International Conference on Turbochargers and Turbocharging, London, UK, 2006, pp. 29-42. doi:10.1016/ B978 - 1 - 84569- 174 - 5.50006- 3.

[15] T. Raitor, W. Neise, Sound generation in centrifugal compressors, Journal of Sound and Vibration 314 (2008) 738 - 756. doi:10.1016/j . jsv.2008.01.034.

[16] N. Figurella, R. Dehner, A. Selamet, K. Tallio, K. Miazgowicz, R. Wade, Noise at the mid to high flow range of a turbocharger compressor, Noise Control Engineering Journal 62 (5) (2014) 306-312. doi: 10.3397/ 1/376229.

[17] A. V. Pai, S. J. Walsh, D. J. O'Boy, R. Chen, Air intake system noise in a turbocharged petrol engine during transient operation, in: INTER-NOISE and NOISE-CON Congress and Conference Proceedings, 2013, pp. 3656-3663.

URL http://WWw.ingentaconnect.com/content/ince/incecp/ 2013/00000247/00000004/art00032
[18] H. Hou, L. Wang, R. Wang, Y. Yang, Effects of bending-torsional ductinduced swirl distortion on aerodynamic performance of a centrifugal compressor, Journal of Thermal Science 26 (2) (2017) 97-106. doi: 10. $1007 /$ s $11630-017-0916-9$.

[19] A. Karim, K. Miazgowicz, B. Lizotte, A. Zouani, Computational aeroacoustics simulation of compressor whoosh noise in automotive turbochargers, SAE Technical Paper (2013-01-1880). doi:10.4271/ 2013- 01 - 1880.

[20] J. Galindo, A. Tiseira, R. Navarro, D. Tarí, C. Meano, Effect of the inlet geometry on performance, surge margin and noise emission of an automotive turbocharger compressor, Applied Thermal Engineering 110 (2017) 875-882. doi:10.1016/j . applthermaleng. 2016.08.099.

[21] A. Torregrosa, A. Broatch, X. Margot, J. García-Tíscar, Y. Narvekar, R. Cheung, Local flow measurements in a turbocharger compressor inlet, Experimental Thermal and Fluid Science 88 (2017) 542-553. doi: 10.1016/j .expthermflusci.2017.07.007.

[22] A. J. Torregrosa, A. Broatch, X. Margot, J. García-Tíscar, Experimental methodology for turbocompressor in-duct noise evaluation based on beamforming wave decomposition, Journal of Sound and Vibration 376 (2016) 60-71. doi : 10.1016/j.jsv. 2016.04.035.

[23] O. Frost, An algorithm for linearly constrained adaptive array processing, Proceedings of the IEEE 60 (8) (1972) 926-935. doi: 10. 1109/PROC. 1972.8817.

[24] C. L. Morfey, Sound transmission and generation in ducts with flow, Journal of Sound and Vibration 14 (1) (1971) 37-55. doi: 10.1016/ $0022-460 \times(71) 90506-2$.

[25] G. Piñero, L. Vergara, J. M. Desantes, A. Broatch, Estimation of velocity fluctuation in internal combustion engine exhaust systems through beamforming techniques, Measurement Science \& Technology 11 (11) (2000) 1585-1595. doi : 10. 1088/0957-0233/11/11/307.

[26] J. Kim, M. Yadav, S. Kim, Characteristics of secondary flow induced by 90-degree elbow in turbulent pipe flow, Engineering Applications of Computational Fluid Mechanics 8 (2) (2014) 229-239. doi : 10 . 1080/ 19942060.2014.11015509.

[27] M. Yang, X. Zheng, Y. Zhang, T. Bamba, H. Tamaki, J. Huenteler, Z. Li, Stability improvement of high-pressure-ratio turbocharger centrifugal compressor by asymmetric flow control-part i: Non-axisymmetrical flow in centrifugal compressor, Journal of Turbomachinery 135 (2) (2013) 021006-1-9. doi:10.1115/1.4006636.

[28] X. Zheng, L. Jin, H. Tamaki, Influence of volute-induced distortion on the performance of a high-pressure-ratio centrifugal compressor with a vaneless diffuser for turbocharger applications, Proceedings of the Institution of Mechanical Engineers, Part A: Journal of Power and Energy 228 (4) (2014) 440-450. doi : 10.1177/0957650913519984.

[29] W. Dean, Note on the motion of fluid in a curved pipe, The London, Edinburgh, and Dublin Philosophical Magazine and Journal of Science 4 (20) (1927) 208-223. doi : 10 . 1080/14786440708564324. 